\title{
Inspired spirals
}

\section{This apparatus, held in London's Science Museum, has some significant purpose - or curiosity value - in the history of physics. Can you guess what it is?}

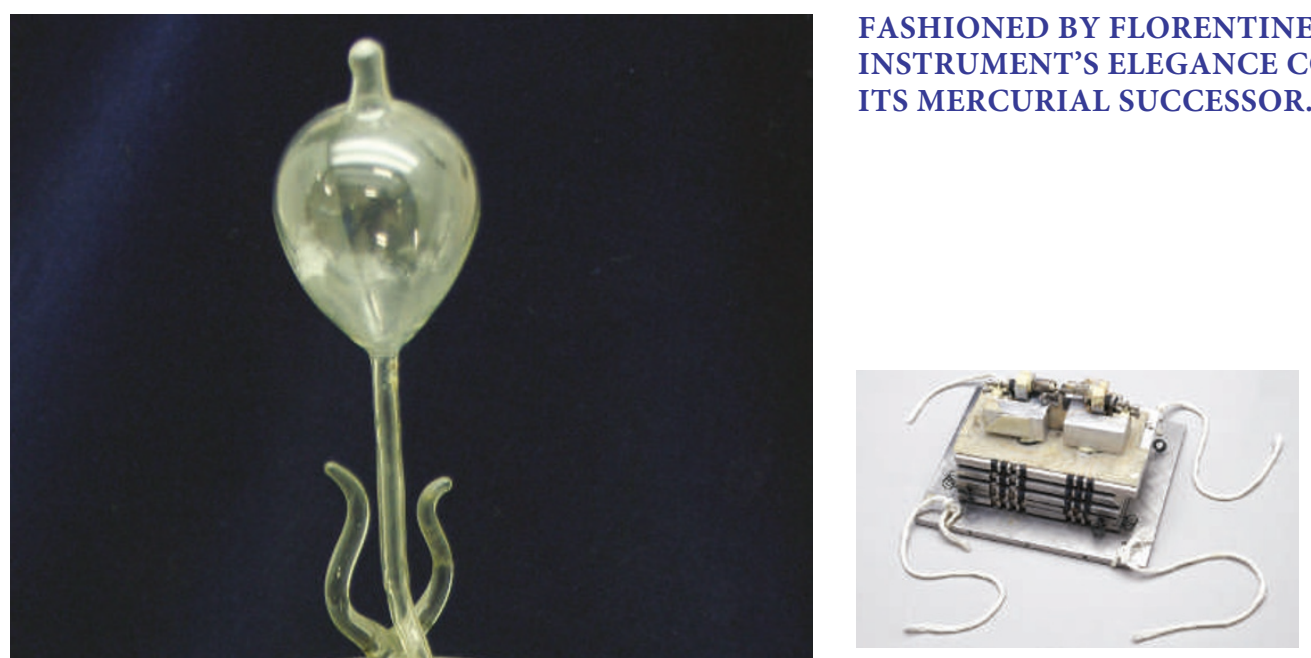

Last month: Atomic force microscope

This is the first atomic force microscope - made in 1985 by Gerd Binnig and Calvin Quate at Stanford University, and Christop Gerber at the IBM San Jose Research Laboratory. It measures topography with a force probe, and, because it does not use lenses, it is the size of the probe rather than diffraction effects that limits the resolution. The AFM also has an advantage over the earlier scanning tunnelling microscope in that it can be used on insulating surfaces.

The components on top form the microscope, the metal plates underneath are for vibrational insulation. The whole apparatus was suspended on cords for further isolation. The sample and probe are attached to two small cylinders, which contain piezoelectric drives for positioning them. The probe is mounted on a tiny cantilever, and the bending of this cantilever is a measure of the force. There are surplus cantilevers and a 'wafer' containing further cantilevers, all made by Quate's group.
David Baselt from Caltech pointed out the capabilities of the AFM in a $1993 \mathrm{PhD}$ thesis. "The atomic force microscope is one of about two dozen types of scanned-proximity probe microscopes. All of these microscopes work by measuring a local property - such as height, optical absorption, or magnetism - with a probe or 'tip' placed very close to the sample. The small probe-sample separation (of the order of the instrument's resolution) makes it possible to take measurements over a small area. To acquire an image, the microscope raster-scans the probe over the sample while measuring the local property in question. The resulting image resembles an image on a television screen in that both consist of many rows or lines of information placed one above the other."

Atomic force microscopy is widely used in a wide range of applications, including medical imaging, mineralogy, drug development and materials research.

\section{JANE WESS}

Jane Wess is a curator at the Science Museum, Exhibition Road, South Kensington, London SW7 2DD, UK.

\section{science museum}

